\title{
The biological functions and mechanism of miR-212 in prostate cancer proliferation, migration and invasion via targeting Engrailed-2
}

\author{
YI ZHOU, ZHIGANG JI, WEIGANG YAN, ZHIEN ZHOU and HANZHONG LI \\ Department of Urology, Peking Union Medical College Hospital, Chinese Academy of Medical Science \\ and Peking Union Medical College, Beijing 100000, P.R. China
}

Received February 9, 2017; Accepted July 6, 2017

DOI: $10.3892 /$ or.2017.5805

\begin{abstract}
Accumulating evidence indicates that Engrailed-2 (EN-2), which is a homeobox-containing transcription factor, act as a candidate oncogene in prostate cancer (PCa). Even though there are some treatments targeting EN-2, however, it is limited because the mechanism of EN-2 upregulation in $\mathrm{PCa}$ cells is still unknown. In this study, we investigate the role of miR-212 on EN-2 expression and explored the mechanism of prostate cancer survival and metastasis. The relative expression levels of miR-212 and EN-2 in PCa samples and adjacent normal tissues as well as in PCa cell lines were detected by using quantitative real-time PCR. CCK-8, TUNEL and Transwell assays were used to analyze cell proliferation, apoptosis and invasion, respectively. EN-2 was identified as a direct target of miR-212 via luciferase reporter and western blot assays. Results showed that the expression level of miR-212 was downregulated in both PCa samples and PCa cell lines when compared with prostate epithelial cells and the adjacent no tumor tissues. Moreover, we found that overexpression of miR-212 suppressed PCa cell proliferation and invasion, promoted PCa cell apoptosis. EN-2 was identified as a direct target gene of miR-212 by using luciferase reporter and western blot assays. Also, the expression of EN-2 and miR-212 in the PCa cells had an opposite correlation. The critical role of miR-212 in inhibiting prostate tumor growth was verified in xenograft models of prostate cancer. These findings highlighted the role of miR-212 in PCa progression. More importantly, we speculate that EN-2 is a direct target gene of miR-212.
\end{abstract}

Correspondence to: Dr Weigang Yan, Department of Urology, Peking Union Medical College Hospital, Chinese Academy of Medical Science and Peking Union Medical College, 1 ShuaiFu Garden, Dongcheng, Beijing 100000, P.R. China

E-mail: bjyanweig@163.com

Abbreviations: PCa, prostate cancer; EN-2, Engrailed-2; PSA, prostate specific antigen

Key words: miR-212, prostate cancer, proliferation, migration, invasion, Engrailed-2

\section{Introduction}

Prostate cancer (PCa) is the second most commonly diagnosed malignancy all over the world, and one of the most common leading cause of cancer-specific mortality in men, which causes 913,000 new cases and >261,000 deaths worldwide each year $(1,2)$. As the development of prostate-specific antigen (PSA) serum level screening and the prostate biopsy through transrectal ultrasound-guided method, the detection rate and incidence of PCa is markedly increased (3). Currently, therapeutic schedules for this disease in a localized stage or when it is still castration-sensitive would have good results in most of the patients. However, such handlings are only palliative in the advanced stage (4). Therefore, new biomarkers and therapeutic targets should be found to get effective treatments for more aggressive stages in prostate cancer.

miRNAs are a kind of small, 20-22 nucleotides non-coding RNAs that regulate the expression of hundreds of target genes through binding to the 3'-untranslated region (3'-UTR) of their target mRNAs (5). miRNAs play critical roles in many kinds of physiological and pathological processes, such as apoptosis, cell proliferation, differentiation, development, stress response and migration (6,7). A growing number of evidence has revealed that miRNAs act as either tumor suppressors or promoters in the process of many kinds of tumors and play important roles in regulating the posttranscriptional gene expression (8-10). miR-212 which is located on chromosome $17 \mathrm{p} 13.3$ and share an identical 5'-seed sequence (11). It has been demonstrated that miR-212 is markedly overexpressed in tongue squamous cell carcinoma (12) and colorectal carcinoma (13), but low-expressed in gastric cancer (14) and non-small cell lung cancers (15). Moreover, studies have shown that dysregulated miR-212 plays a critical role in the proliferation and invasion of many solid malignancies, which makes it a potential target for tumor treatment (16). However, the exact function of miR-212 in PCa has not yet been studied.

Engrailed-2 (EN-2), which is a homeodomain-containing transcription factor, was recently verified to be involved in the development of prostate cancer (17) and is also confirmed to express abnormally in prostate cancer cell lines (18). Recent studies had shown that EN-2 is a potential biomarker for pros- 
tate cancer $(19,20)$. While the regulatory mechanism and the role in progress of prostate cancer still almost known.

In this study, we aimed to detect the expression of miR-212 in PCa tissues and cell lines as well as to illustrate the role of miR-212 in PCa cell line proliferation, colony formation and migration. Importantly, we also illustrate EN-2 as a potential target gene of miR-212.

\section{Materials and methods}

Collection and cell lines. Prostate cancer samples and the adjacent normal tissues were collected from 58 patients who underwent tumor-resection surgery in Peking Union Medical College Hospital from September 1, 2014 to January 1, 2016. Patients signed an informed consent form. The tissues were separately frozen in liquid nitrogen for further study. Protocols were approved by the institutional ethics committee of Peking Union Medical College Hospital and carried out in accordance with the guidelines for ethical management. PCa cell lines, PC3 and DU145, and prostate epithelial cells were purchased from American Type Culture Collection (ATCC, USA). PC3 was incubated in F-12 medium, and DU145 was grown in DMEM medium (Life Technologies, Inc., USA), and all the mediums were supplemented with penicillin/streptomycin (100 U/ml and $100 \mathrm{mg} / \mathrm{ml}$, respectively) and 10\% fetal bovine serum (Life Technologies Inc.). The human prostate epithelial cells (hPrEC) were cultured in prostate epithelium basal media. All the cells were incubated at $37^{\circ} \mathrm{C}$ in a humidified atmosphere of $5 \% \mathrm{CO}_{2}$.

Quantitative real-time PCR. Total RNA was extracted from the tissues or cells via TRIzol reagent (Invitrogen, Carlsbad, CA, USA) according to the manufacturer's instructions and quantified with UV absorbancy at 260 and $280 \mathrm{~nm}$ (A260/280). Subsequently the RNA was reverse-transcribed into cDNA by using reverse transcription system (Thermo Scientific, CA, USA). The expression level of miR-212 was detected by the Applied Biosystems 7500 Real-time PCR system (ABI) using the TaqMan MicroRNA assay kits (Applied Biosystems, CA, USA). U6 was used as the control normalizer. The gene expression of EN-2 was also analyzed by SYBR Green and normalized with GAPDH. The specificity of primer sequences was analyzed by dissociation curve, $2^{-\Delta \Delta \mathrm{Ct}}$ (cycle threshold) was used to calculate the relative gene expression levels.

Western blotting. Protein from tissues or cells was collected by using RIPA buffer which contain a protease inhibitor cocktail and phosphatase inhibitors (Sigma, St. Louis, MO, USA), according to the manufacturer's instructions and quantified via bicinchoninic acid (BCA) kit (Thermo Scientific). Protein samples $(30 \mu \mathrm{g})$ were separated by SDS-PAGE and then transferred onto the polyvinylidene difluoride (PVDF, Millipore, Bedford, MA, USA) membranes. Western blotting was performed using anti- $\beta$-actin, anti-EN-2, anti-Bcl-2 and anti-Bax (CST, Denver, CO, USA). The protein levels were detected with an ECL kit (Thermo Scientific) according to the manufacturer's instructions.

CCK-8 assay measurements of the proliferation of PCa cells. CCK-8 assay was performed to evaluate cell proliferation according to the manufacturer's instructions. For experiments the cells were seeded at a density of $5 \times 10^{3}$ cells per well in 96 -well plates and cultured for various periods of time $(0,24$, 48 and $72 \mathrm{~h}$ ). The absorbance at $450 \mathrm{~nm}$ was measured using an electroluminescence immunosorbent assay reader (Thermo Fisher Scientific, Waltham, MA, USA).

Apoptosis of PCa cells were detected by flow cytometry. Cells were collected and washed twice with cold phosphate-buffered saline solution (PBS) to remove floating cells then labeled with Annexin V-FITC (BD Biosciences, San Jose, CA, USA). Apoptosis were evaluated with a flow cytometry analyzer. Data were analyzed by Flowjo 7.6 software.

TUNEL detection of apoptosis of PCa cells. Cells were fixed with $4 \%$ paraformaldehyde, permeabilized in $0.2 \%$ Triton X-100, and incubated with the TUNEL detection kit from Roche (Roche, Shanghai, China) at $37^{\circ} \mathrm{C}$ for $1 \mathrm{~h}$. The samples were mounted in mounting media containing DAPI. Fluorescent images were captured using a fluorescence microscope. The total number of DAPI positive cells and total number of TUNEL positive cells were counted.

Cell invasion assays. Cell invasion assays were performed by using 24-well Transwell plates (BD Biocoat, USA). Cells transfected with the miR-212 mimics and mimic control for $24 \mathrm{~h}$. Then the cells were plated onto the 24-well upper chamber with a membrane that was pre-treated with Matrigel (100 $\mu \mathrm{g}$ per well; BD Biosciences). In the lower portion of the chamber, fresh medium containing $10 \%$ FBS was added. After the cells were incubated for $24 \mathrm{~h}$ at $37^{\circ} \mathrm{C}$, the cells in the upper chamber were removed. Invaded cells were fixed with $4 \%$ formaldehyde, stained with $0.5 \%$ crystal violet, and counted under a microscope.

Wound healing assay. Cells were seeded into 6-well plates. Wounds were created in the confluent cell monolayer using a $200-\mu 1$ sterile pipette tip, and the other free-floating cells and debris were removed by washing with PBS three times. Medium was then added, and the culture plates were incubated at $37^{\circ} \mathrm{C}$ for another 24 or $48 \mathrm{~h}$. Wound healing within the scraped wound line was measured at a 48-h time-point by using microscope (Nikon, Tokyo, Japan).

Dual luciferase reporter assay. TargetScan software (http:// www.targetscan.org/) was used to identify the potential target gene of miR-212. The putative miR-212 binding sites or mutant at the 3'-UTR of EN-2 were cloned into a vector and EN-2 3'-UTR WT or EN-2 3'-UTR Mut, was generated, respectively. EN-2 3'-UTR WT or EN-2 3'-UTR Mut was transfected into PCa cells which stably overexpressed miR-212 and transfected with negative control. Dual-Luciferase Reporter assay system (Promega, Madison, WI, USA) was used to detect the luciferase activity $36 \mathrm{~h}$ after transfection.

Lentivirus production and transfection. The LV-miR-212 mimics and mimics control were purchased from Gene-Chem (Shanghai, China). Cells were seed into 24 -well plates at a density of $30 \%$ and cultured overnight before transfection. Cells were transfected with miR-212 mimics or mimics control 

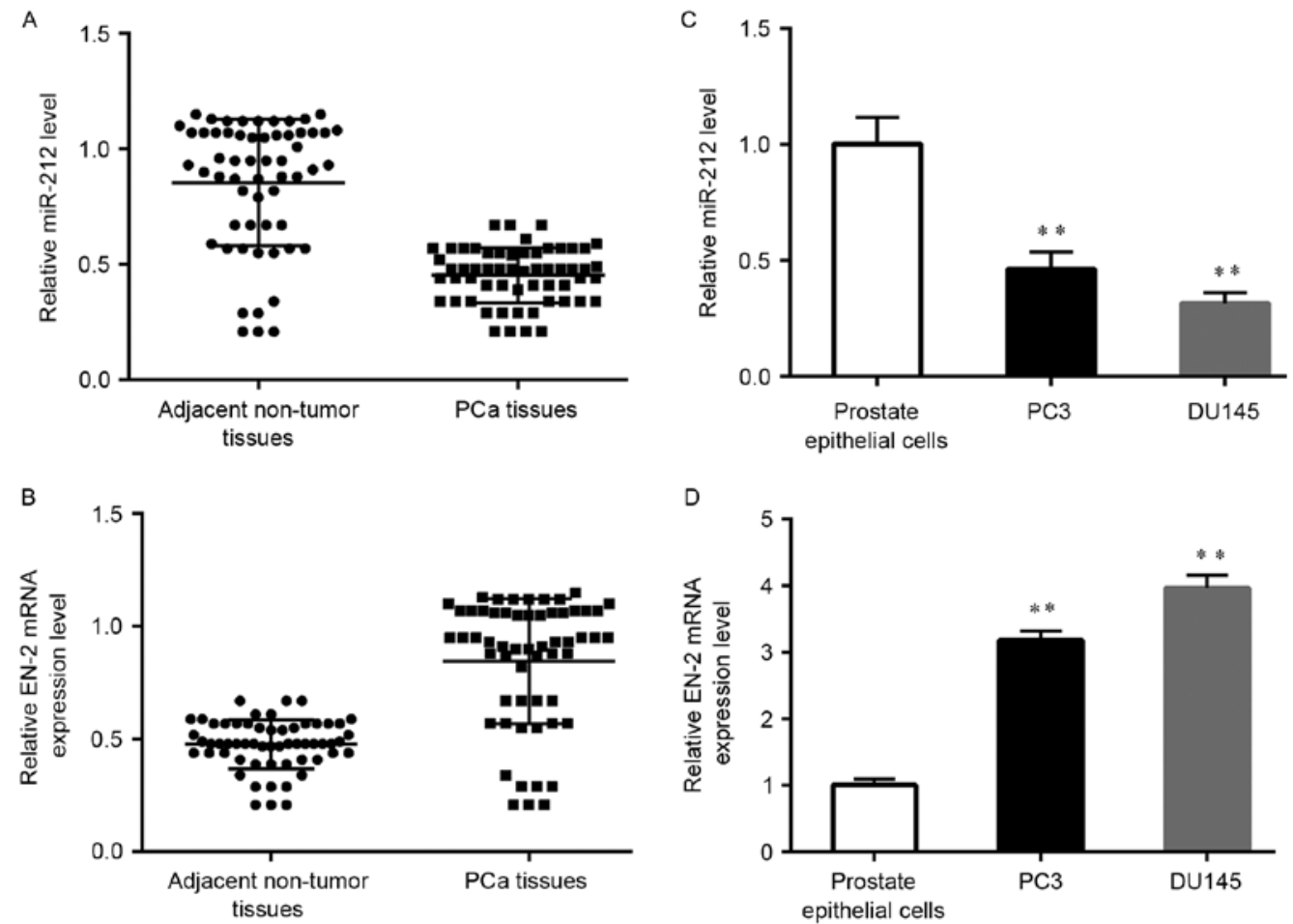

Figure 1. The expression of miR-212 and EN-2 in clinical specimens and PCa cell lines. (A) The level of miR-212 was downregulated in PCa tissues compared to the adjacent non-tumor tissues. (B) EN-2 in PCa tissues was markedly higher than in the adjacent non-tumor tissues. (C) miR-212 was dramatically decreased in PCa cell linescompared to the prostate epithelial cells ( $\left.{ }^{* *} \mathrm{P}<0.01\right)$. (D) EN-2 was significantly increased in PCa cell lines compared to the prostate epithelial cells $\left({ }^{* *} \mathrm{P}<0.01\right)$.

by using Lipofectamine 2000 reagent (Invitrogen) according to the manufacturer's protocols.

Animals and xenograft. In this study all the animal procedures and experiments were performed in accordance with the National Institutes of Health Guide for Care and Use of Laboratory Animals. The 6-8-week-old nude male mice [BALB/c A-nu (nu/nu)] were purchased from Shanghai SLAC Laboratory Animal Co., Ltd. (Shanghai, China) and housed in the Animal Resource Facility at Laboratory Animal Centre. Cells stably expressing either miR-212 overexpression (LV-miR-212) or miRNA control were injected subcutaneously into the mice. The volume of the tumor was recorded for 7 weeks (once each week).

Statistical analysis. All quantitative data presented are the mean \pm SD from at least three independent experiments. The relationship between the expression level of miR-212 and clinicopathological parameters was examined using Fisher's exact test or $\chi^{2}$ test. One-way ANOVA test or Student's t-test was used to analyze the other date. The significant statistical differences level was set at $\mathrm{P}<0.05$. SPSS 18.0 and Graph Pad Prism 6.0 software were used for date analysis.

\section{Results}

The expression level of miR-212 and EN-2 in human $\mathrm{PCa}$ tissues and PCa cell lines. In order to confirm the molecular mechanisms underlying $\mathrm{PCa}$, we analyzed the expression level of miR-212 and EN-2 in a series of clinical PCa tissues. Results showed that miR-212 was markedly decreased in
Table I. The relationship between miR-212 expression level and clinicopathological features in our PCa cases.

\begin{tabular}{lrrrr}
\hline Parameters & N (case) & Low (\%) & High (\%) & P-value \\
\hline Age & & & & 0.168 \\
$<65$ & 13 & $7(53.8)$ & $6(46.2)$ & \\
$\geq 65$ & 45 & $21(46.7)$ & $24(53.3)$ & \\
Gleason scores & & & & 0.005 \\
$<7$ & 11 & $3(27.3)$ & $8(72.7)$ & \\
$\quad 7$ & 17 & $8(47.1)$ & $9(52.9)$ & \\
$>7$ & 30 & $21(70.0)$ & $9(30.0)$ & \\
Tumor stage & & & & 0.067 \\
$\leq$ T2b & 43 & $21(48.8)$ & $22(51.2)$ & \\
$\geq$ T3b & 15 & $4(26.7)$ & $11(73.3)$ & \\
Preoperative PSA & & & & \\
level (ng/ml) & & & & 0.076 \\
$\leq 10$ & 11 & $8(72.7)$ & $3(27.3)$ & \\
$>10$ & 47 & $20(42.6)$ & $27(57.4)$ & \\
Distant metastasis & & & & 0.002 \\
M0 & 45 & $28(62.2)$ & $17(37.8)$ & \\
M1 & 13 & $2(15.4)$ & $11(84.6)$ & \\
\hline
\end{tabular}

$\mathrm{PCa}$, prostate cancer, PSA, prostate specific antigen.

cancer samples when compared with the adjacent normal tissues (Fig. 1A). In contrary, the level of EN-2 mRNA was 


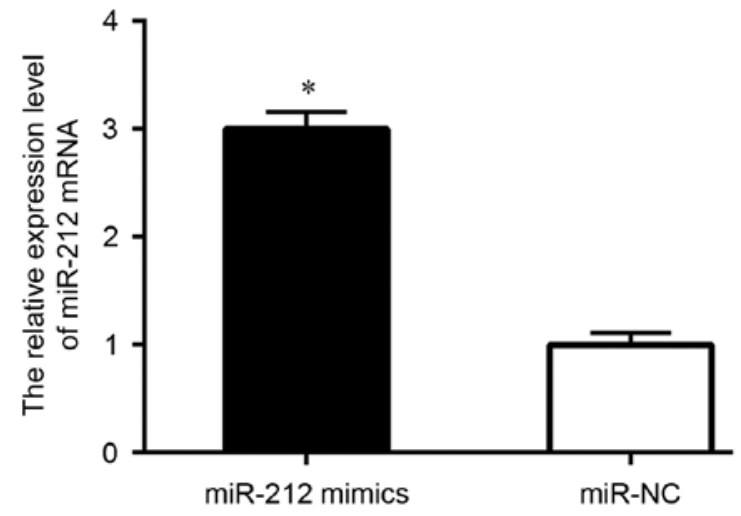

Figure 2. The expression level of miR-212 in PCa cell lines transfected with miR-NC, miR-212 mimics. The miR-212 mRNA level was significantly increased in miR-212 mimics when compared with the miR-NC ( $\left.{ }^{*} \mathrm{P}<0.05\right)$. over-expressed (Fig. 1B). Subsequently, we measured the miR-212 and EN-2 level in PCa cell lines and the human prostate epithelial cells (hPrEC) by RT-qPCR. As shown in Fig. 1C, the expression level of miR-212 in PCa cells was significantly lower than that in hPrEC cells. While the expression level of EN-2 had the opposite trend (Fig. 1D). The relationship between miR-212 expression level and clinicopathological features in our PCa cases is presented in Table I. The decreased miR-212 was significantly associated with high Gleason scores $(\mathrm{P}<0.05)$. As shown in Table I, the decreased levels of miR-212 was present in 3 of 11 (27.3\%) cases with low Gleason score $(<7), 8$ of $17(47.1 \%)$ cases with Gleason score 7 , but 21 of $30(70.0 \%)$ cases with high Gleason score $(>7)$. The expression level of miR-212 was obviously decreased in $\mathrm{PCa}$ patients with distant metastasis $(\mathrm{P}<0.05)$. There were
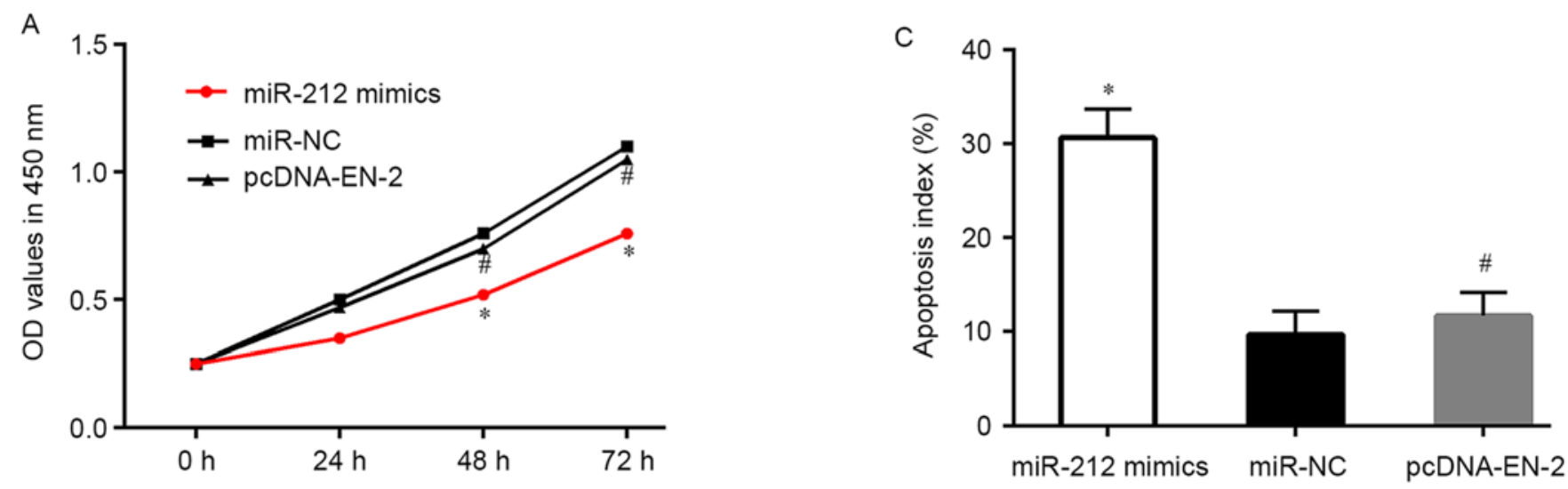

B
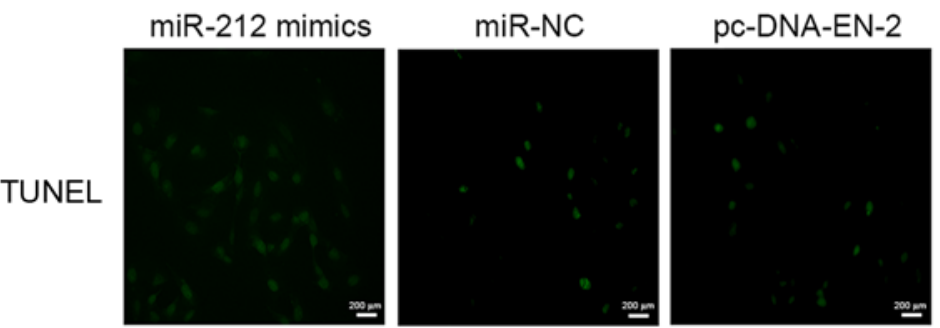

D
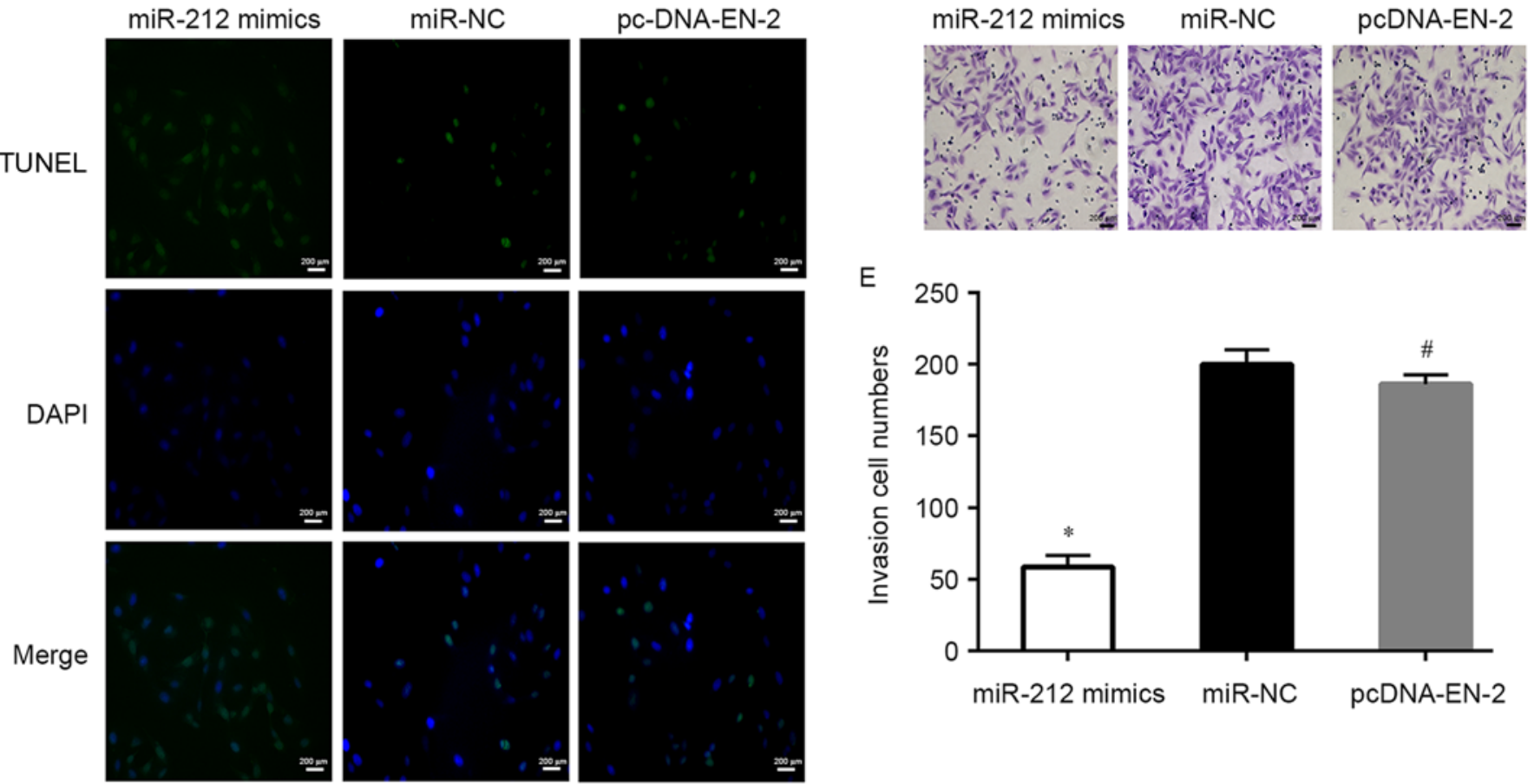

Figure 3. miR-212 inhibits PCa cell proliferation, invasion and promotes apoptosis. (A) CCK-8 assay showed that miR-212 could inhibit the proliferation of PCa cells, recovery of EN-2 promoted PCa cell proliferation ( ${ }^{*} \mathrm{P}<0.05$ when compared with the miR-NC, ${ }^{*} \mathrm{P}<0.05$ when compared with the miR-212 mimics). (B and C) TUNEL assay revealed that the apoptosis of PCa cells increased in miR-212 mimics when compared with the miR-NC ("P<0.05), apoptosis of PCa cells markedly decreased in EN-2 restoration $\left({ }^{*} \mathrm{P}<0.05\right.$ when compared with the miR-212 mimics). (D and E) The invasion ability of PCa cells decreased in upregulation of miR-212 ( ${ }^{*} \mathrm{P}<0.05$ when compared with the miR-NC), recovery of EN-2 promoted the invasion ability $\left({ }^{*} \mathrm{P}<0.05\right.$ when compared with the miR-212 mimics). 


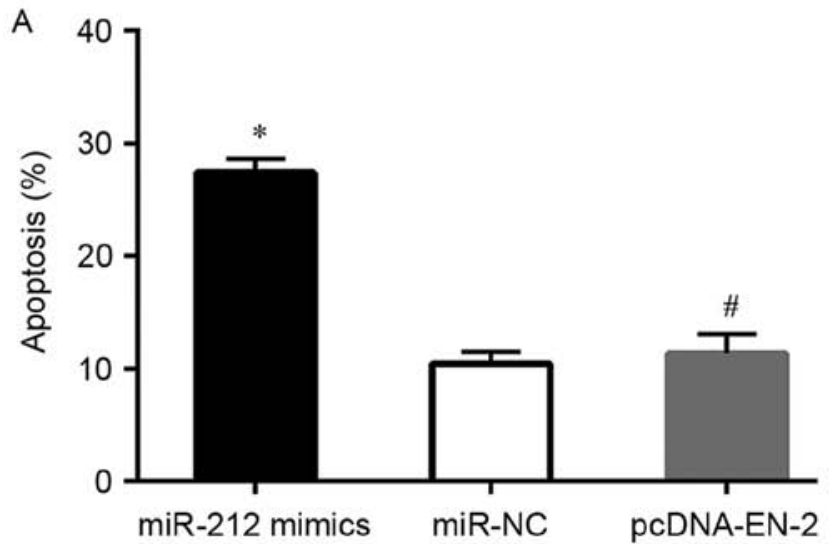

B

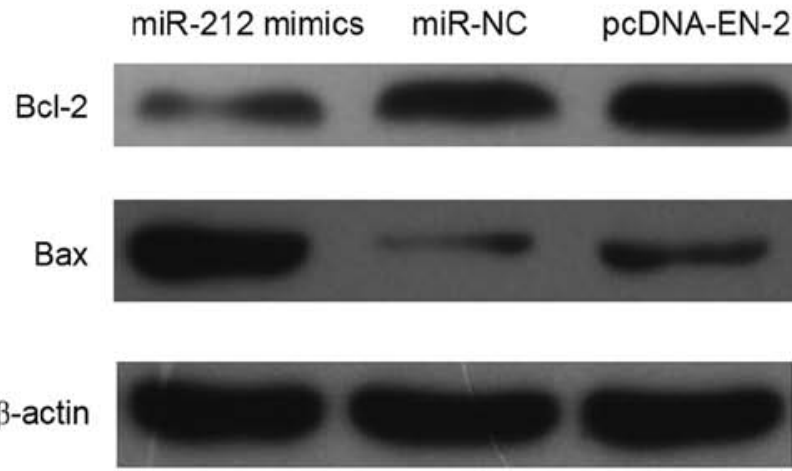

Figure 4. miR-212 induces apoptosis of PCa cells. (A) Flow cytometry showed that apoptosis of PCa cells increased after miR-212 upregulation ("P<0.05 when compared with the miR-NC), and restoration of EN-2 inhibited apoptosis $\left({ }^{*} \mathrm{P}<0.05\right.$ when compared with the miR-212 mimics). (B) The protein expression levels of Bcl-2 and Bax.

A

miR-212 mimics

$\mathrm{Oh}$

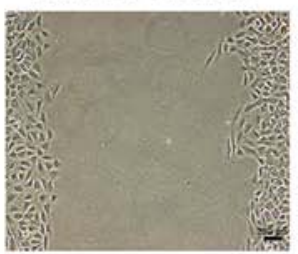

$48 \mathrm{~h}$

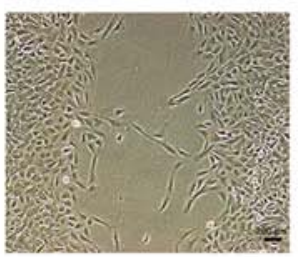

miR-NC
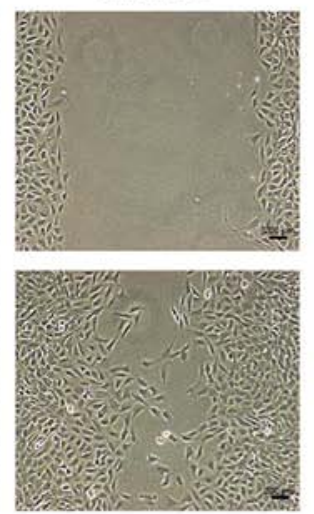

PCDNA-EN-2

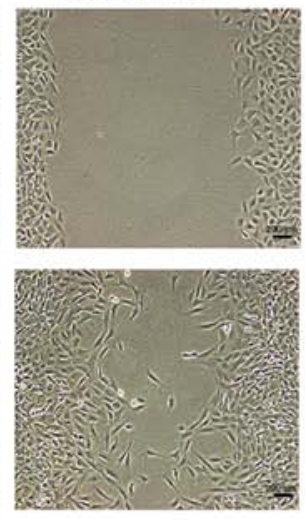

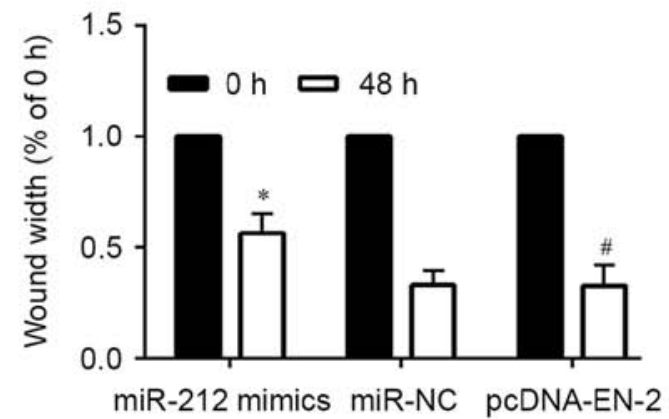

Figure 5. miR-212 inhibits PCa cell migration. Wound healing assay demonstrated that upregulation of miR-212 significantly inhibited PCa cell migration $($ ( $\mathrm{P}<0.05$ when compared with the miR-NC), recovery of EN-2 promoted PCa cell migration $(" \mathrm{P}<0.05$ when compared with the miR-212 mimics).

no statistically significant difference between miR-212 level and the age of the patients, PSA levels or tumor stage.

Upregulation of miR-212 inhibits PCa cell survival, proliferation, invasion and migration. Next, in order to verify the role of miR-212 in control PCa cell survival and progress, miR-212 mimics and miR-NC were transfected into DU145 cells, then cells were collected for analysis. The level of miR-212 was detected by qRT-PCR. Results showed that the expression level of miR-212 in the mimic group was markedly increased when compared with the control (Fig. 2). CCK-8 assay demonstrated that miR-212 upregulation significantly suppressed DU145 cells proliferation (Fig. 3A). Moreover, the TUNEL and flow cytometry assays demonstrated that the apoptosis of DU145 cells was increased when transfected with miR-212 mimics (Figs. 3B and $\mathrm{C}$ and 4A), whereas, the protein expression levels of Bcl-2 decreased and Bax markedly increased in DU145 cells after transfected with miR-212 mimics (Fig. 4B). Subsequently, we measured the effect of miR-212 on cell invasion, the Transwell assays indicated that miR-212 mimics transfected DU145 cells markedly suppressed cell invasion ability when compared to the mimic control cells (Fig. 3D and E). A similar tendency was discovered in the wound healing assays (Fig. 5). These outcomes clearly suggested that restoration of the expression of miR-212 inhibits PCa cell proliferation, invasion and migration.

miR-212 decreases EN-2 expression by binding to 3'-UTR of its $m R N A$. In order to illustrate that EN-2 is a direct target of miR-212, EN-2 wild-type (WT) or mutant 3'-UTR (Mut) (Fig. 6A) were subcloned into a luciferase reporter vector and co-transfected with miR-212 mimics or negative control (NC) into PCa cells. Then, luciferase activity was detected after 48-h transfection. The results demonstrated that miR-212 markedly inhibited luciferase activity of the EN-2 WT 3'-UTR but had no influence on the mutant (Fig. 6B). To verify whether miR-212 can regulate the expression of EN-2, miR-212 mimics was transfected into DU145 cells for analysis. Results showed that the mRNA and protein level of EN-2 was markedly suppressed by miR-212 upregulation (Figs. 6C-E and 8).

EN-2 re-introduction reverses the anti-cell survival and metastasis role of miR-212. In order to study the effect of 
A

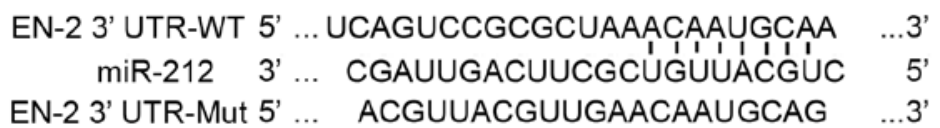

B

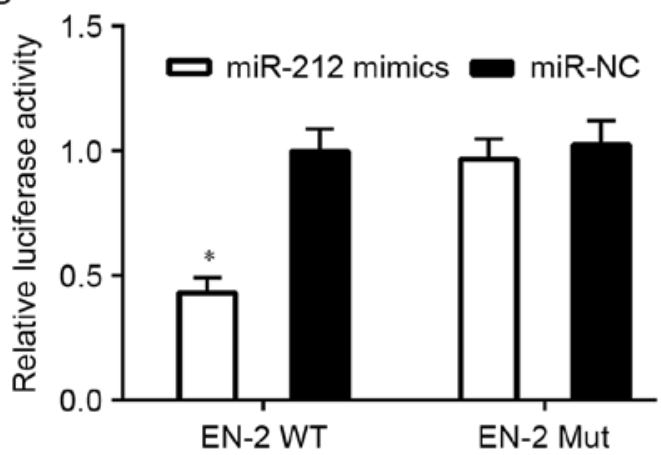

D

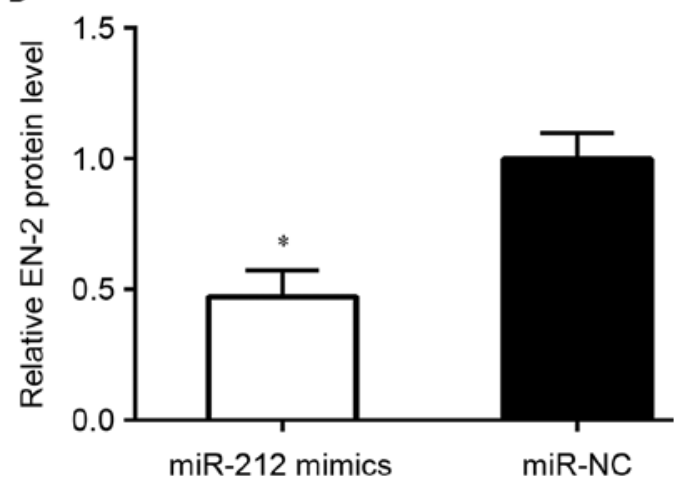

C

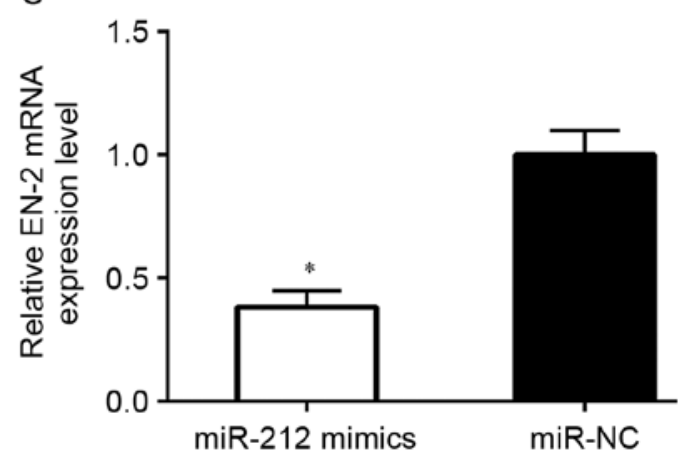

E

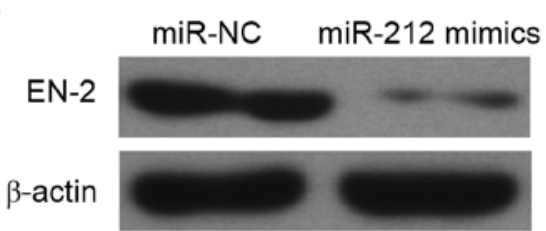

Figure 6. EN-2 is a direct target of miR-212. (A) Sequence of miR-212 and the EN-2 3'-UTR, which contains a predicted miR-212 biding site. (B) Luciferase assay in PCa cells co-transfected with miR-212 mimics and mimic control containing the EN-2 3'-UTR (WT) or a mutant (Mut) ('P<0.05 when compared with the EN-2 Mut). (C and E) miR-212 transfection suppressed the EN-2 protein and gene levels ( $\mathrm{P}<0.05$ when compared with the miR-NC).

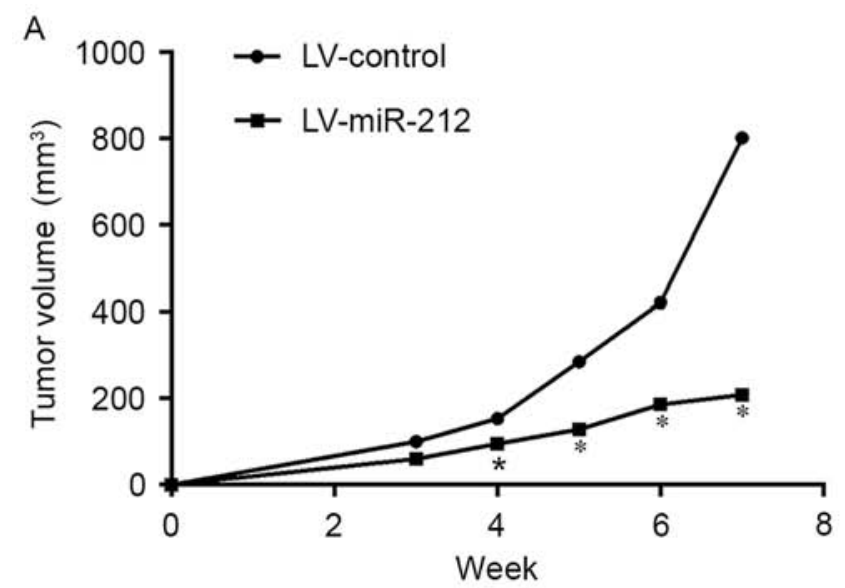

B

LV-control

LV-miR-212

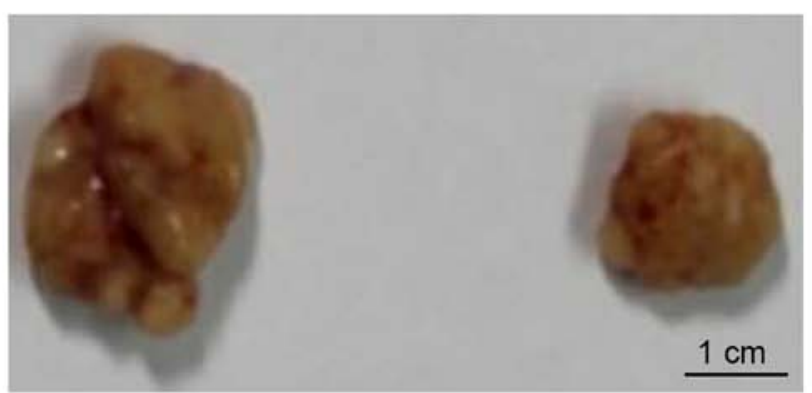

Figure 7. miR-212 suppresses PCa growth in vivo. (A and B) Stable transfection of PCa cells with miR-212 reduced the tumor size ("P<0.05 when compared with the LV-control).

EN-2 in PCa survival and invasion, miR-212 mimics and pcDNA-EN-2 were co-transfected into PCa cell lines, and after transfected for $48 \mathrm{~h}$, cell survival and the ability of invasion were measured. Results showed that restoration of EN-2 reversed the anti-cell survival actions of miR-212 and the cell proliferation ability also recovered in comparison to miR-212 mimics (Figs. 3A-C and 4A). Moreover, the capability of invasion of PCa cells was significantly restored via upregulation of EN-2 when compared with miR-212 mimics (Fig. 3D and E), the migration ability of PCa cells was markedly increased by EN-2 restoration when compared with miR-212 mimics (Fig. 5). 


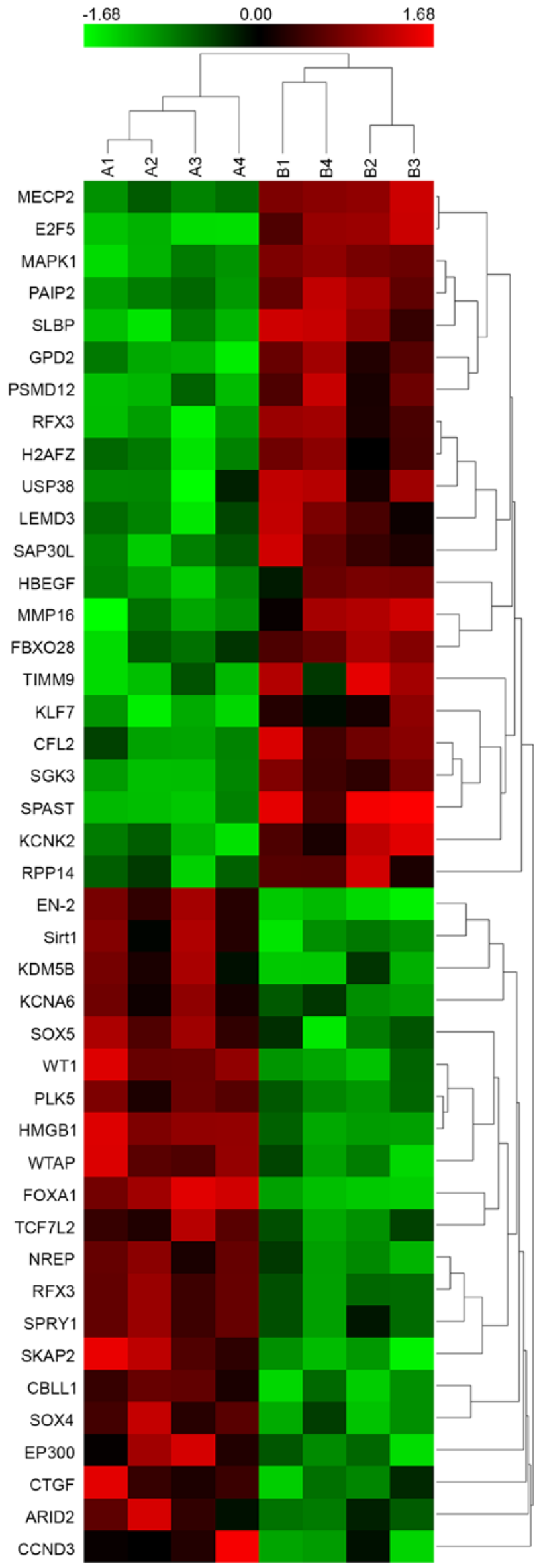

Figure 8. Overexpression of miR-212 significantly inhibits the level of EN-2. The green indicates decreased, and red increased (A1-A4 indicate miR-NC, B1-B4 indicate miR-212 mimics).
Upregulation of miR-212 suppresses PCa growth in vivo. In order to confirm the role of miR-212 against tumor growth in vivo, an animal model was made by nude mice subcutaneously injected with LV-miR-212. The tumor volume of subcutaneous xenograft tumors in nude mice apparently decreased with miR-212 upregulation when compared with the LV-control and the significant difference emerged from 4-week post-tumor transplantation (Fig. 7).

\section{Discussion}

$\mathrm{PCa}$ is the most frequently diagnosed malignance in men, and it is the second cause of tumor-related death in developed countries $(1,21)$. Therefore, the early diagnosis is of great importance to allow successful treatment. Currently, the main methods for PCa diagnosis are PSA testing, digital rectal examination (DRE), PCA3, multiparametric magnetic resonance image (mpMRI) and Gleason score (22). However, the therapeutic approaches are relatively limited. miRNAs are a series of small non-coding RNAs that are widely involved in both physiological and pathological condition (23-25). For example, miR-135a and miR-21 are separately taken part in the cancer pathological process and insulin resistance $(26,27)$. At the same time, there are studies also reporting that miR-125b and miR-449a are respectively, participating in the physiological condition of apoptosis and autophagy $(28,29)$. It has been widely spread that dysregulation of miRNAs may cause cells dysfunction and further give rise to oncogenesis (30). Therefore, research on miRNAs may illustrate the understanding of PCa development and be a great help for recognizing potential biomarkers.

miR-212 is one of the most important miRNAs that has been studied in many kinds of cancers, including hepatocellular carcinoma and pancreatic cancer $(31,32)$. In our study, we firstly investigated the expression level of miR-212 in PCa patients and found that it was markedly decreased in PCa patient tissues than in the adjacent normal tissues. In PCa cell lines, the level of miR-212 also decreased compared to the hPrEC. Importantly, the low level of miR-212 has a significant correlation with high Gleason score and distant metastasis. These results suggested that miR-212 play an important role in the pathogenesis of $\mathrm{PCa}$.

Previous research has revealed that miR-212 negatively regulates starvation-induced autophagy in PCa cells by inhibiting SIRT1, upregulation of miR-212 also leads to suppression of angiogenesis and cellular senescence (33). In this study, overexpression of miR-212 significantly suppressed cell proliferation, invasion and migration, and markedly promoted apoptosis of PCa cells. Bcl-2 and Bax are apoptosis-related genes. In this study, we found that upregulation of miR-212, regulates Bcl-2 family members, the level of Bax increased and the Bcl-2 decreased, while the recovery of EN-2 promoted the $\mathrm{Bcl}-2$ resistance to apoptosis and inhibited Bax promoting apoptosis. Which was consistent with the above previous study. In order to further research the function of miR-212 in PCa cells, we detected the role of miR-212 in the growth of subcutaneous xenograft tumors in nude mice. In accordance with the in vitro study, upregulation of the expression of miR-212 suppressed the growth of xenograft tumors in vivo. Collectively, these results sustained the concept that miR-212 may act as a tumor suppressor in $\mathrm{PCa}$. 
Many target genes for miR-212 have been found in various kinds of malignancies such as Lin28B in PCa (34). There are also some other targets for miR-212, including RB, SMAD2, FOXA1 and SGK3 have been reported in some other cancers $(11,32,35,36)$. Although the role of miR-212 has been studied in many tumors, its mechanistic role in $\mathrm{PCa}$ is still unclear. Increasing evidence has recognized that EN-2 acts as a novel well-known biomarker for diagnosing PCa $(37,38)$, while its effect on PCa oncogenesis has not been defined. There are also studies demonstrating that the expression of EN-2 dysregulated in many human cancers, and inhibiting EN-2 expression can suppress the development of bladder cancer and chronic myelogenous leukemia $(39,40)$, which suggested that EN-2 play an important role in the progression of cancer. In order to study the involvement of EN-2 and the role of interaction between miR-212 and EN-2 in oncogenesis of PCa, we studied PCa cell activity in DU145 cells co-transfected with miR-212 mimics and pcDNAEN-2. Results demonstrated that EN-2 restoration effectively reversed repressive effect of miR-212 on cell survival and progression. In this study, we also confirmed that EN-2 was as a direct target gene of miR-212 in PCa cells.

As demonstrated above, the level of miR-212 was downregulated in $\mathrm{PCa}$ patients and the cell lines. Upregulation of miR-212 markedly suppressed PCa cell proliferation and promoted apoptosis and it may be via targeting EN-2. Our findings introduced a better understanding of miR-212 in $\mathrm{PCa}$ progression, which is likely to provide more precise targets for diagnostic and therapeutics against $\mathrm{PCa}$, and we will carry out further study to confirm this suggestion.

\section{References}

1. Siegel RL, Miller KD and Jemal A: Cancer statistics, 2015. CA Cancer J Clin 65: 5-29, 2015.

2. Ferlay J, Shin HR, Bray F, Forman D, Mathers C and Parkin DM: Estimates of worldwide burden of cancer in 2008: GLOBOCAN 2008. Int J Cancer 127: 2893-2917, 2010.

3. Cuzick J, Thorat MA, Andriole G, Brawley OW, Brown PH, Culig Z, Eeles RA, Ford LG, Hamdy FC, Holmberg L, et al: Prevention and early detection of prostate cancer. Lancet Oncol 15: e484-e492, 2014.

4. Chen FZ and Zhao XK: Prostate cancer: Current treatment and prevention strategies. Iran Red Crescent Med J 15: 279-284, 2013.

5. Bartel DP: MicroRNAs: Target recognition and regulatory functions. Cell 136: 215-233, 2009.

6. Steinfeld I, Navon R, Ach R and Yakhini Z: miRNA target enrichment analysis reveals directly active miRNAs in health and disease. Nucleic Acids Res 41: e45, 2013.

7. Medina PP and Slack FJ: microRNAs and cancer: An overview. Cell Cycle 7: 2485-2492, 2008.

8. van Kouwenhove M, Kedde M and Agami R: MicroRNA regulation by RNA-binding proteins and its implications for cancer. Nat Rev Cancer 11: 644-656, 2011.

9. Calin GA and Croce CM: MicroRNA signatures in human cancers. Nat Rev Cancer 6: 857-866, 2006.

10. Dalmay T: Mechanism of miRNA-mediated repression of mRNA translation. Essays Biochem 54: 29-38, 2013.

11. Park JK, Henry JC, Jiang J, Esau C, Gusev Y, Lerner MR, Postier RG, Brackett DJ and Schmittgen TD: miR-132 and miR-212 are increased in pancreatic cancer and target the retinoblastoma tumor suppressor. Biochem Biophys Res Commun 406: 518-523, 2011.

12. Scapoli L, Palmieri A, Lo Muzio L, Pezzetti F, Rubini C, Girardi A, Farinella F, Mazzotta M and Carinci F: MicroRNA expression profiling of oral carcinoma identifies new markers of tumor progression. Int J Immunopathol Pharmacol 23 1229-1234, 2010.
13. Schetter AJ, Leung SY, Sohn JJ, Zanetti KA, Bowman ED, Yanaihara N, Yuen ST, Chan TL, Kwong DL, Au GK, et al: MicroRNA expression profiles associated with prognosis and therapeutic outcome in colon adenocarcinoma. JAMA 299: 425-436, 2008.

14. Wada R, Akiyama Y, Hashimoto Y, Fukamachi H and Yuasa Y: miR-212 is downregulated and suppresses methyl-CpG-binding protein MeCP2 in human gastric cancer. Int J Cancer 127: 1106-1114, 2010.

15. Incoronato M, Garofalo M, Urso L, Romano G, Quintavalle C, Zanca C, Iaboni M,Nuovo G,Croce CM and Condorelli G: miR-212 increases tumor necrosis factor-related apoptosis-inducing ligand sensitivity in non-small cell lung cancer by targeting the antiapoptotic protein PED. Cancer Res 70: 3638-3646, 2010.

16. Mehta A, Mann M, Zhao JL, Marinov GK, Majumdar D, Garcia-Flores Y, Du X, Erikci E, Chowdhury K and Baltimore D: The microRNA-212/132 cluster regulates B cell development by targeting Sox4. J Exp Med 212: 1679-1692, 2015.

17. Morgan R, Boxall A, Bhatt A, Bailey M, Hindley R, Langley S, Whitaker HC, Neal DE, Ismail M, Whitaker H, et al: Engrailed-2 (EN2): A tumor specific urinary biomarker for the early diagnosis of prostate cancer. Clin Cancer Res 17: 1090-1098, 2011.

18. Bose SK, Bullard RS and Donald CD: Oncogenic role of engrailed-2 (en-2) in prostate cancer cell growth and survival. Transl Oncogenomics 3: 37-43, 2008.

19. Marszałł MP, Sroka W, Adamowski M, Słupski P, Jarzemski P, Siódmiak J and Odrowąż-Sypniewska G: Engrailed-2 protein as a potential urinary prostate cancer biomarker: A comparison study before and after digital rectal examination. Eur J Cancer Prev 24: 51-56, 2015.

20. McGrath SE, Michael A, Morgan R and Pandha H: EN2: A novel prostate cancer biomarker. Biomarkers Med 7: 893-901, 2013.

21. Ferlay J, Steliarova-Foucher E, Lortet-Tieulent J, Rosso S, Coebergh JW, Comber H, Forman D and Bray F: Cancer incidence and mortality patterns in Europe: Estimates for 40 countries in 2012. Eur J Cancer 49: 1374-1403, 2013.

22. Bertoli G, Cava C and Castiglioni I: MicroRNAs as biomarkers for diagnosis, prognosis and theranostics in prostate cancer. Int J Mol Sci 17: 421, 2016.

23. Kloosterman WP and Plasterk RH: The diverse functions of microRNAs in animal development and disease. Dev Cell 11: 441-450, 2006.

24. Ha TY: MicroRNAs in human diseases: From cancer to cardiovascular disease. Immune Netw 11: 135-154, 2011.

25. Baranwal S and Alahari SK: miRNA control of tumor cell invasion and metastasis. Int J Cancer 126: 1283-1290, 2010.

26. Zhang C, Chen X, Chen X, Wang X, Ji A, Jiang L, Sang F and Li F: miR-135a acts as a tumor suppressor in gastric cancer in part by targeting KIFC1. Onco Targets Ther 9: 3555-3563, 2016.

27. Zhao XY and Shao K: Roles of MicroRNA-21 in the pathogenesis of insulin resistance and diabetic mellitus-induced non-alcoholic fatty liver disease. Zhongguo Yi Xue Ke Xue Yuan Xue Bao 38: 144-149, 2016 (In Chinese).

28. Yu G, Zhan X, Zhang Z and Li Y: Overexpression of miR-125b promotes apoptosis of macrophages. Xi Bao Yu Fen Zi Mian Yi Xue Za Zhi 32: 958-962, 2016 (In Chinese).

29. Han R, Ji X, Rong R, Li Y, Yao W, Yuan J, Wu Q, Yang J, Yan W, Han L, et al: miR-449a regulates autophagy to inhibit silicainduced pulmonary fibrosis through targeting Bcl2. J Mol Med (Berl) 94: 1267-1279, 2016.

30. Bouyssou JM, Manier S, Huynh D, Issa S, Roccaro AM and Ghobrial IM: Regulation of microRNAs in cancer metastasis. Biochim Biophys Acta 1845: 255-265, 2014.

31. Ma C, Nong K, Wu B, Dong B, Bai Y, Zhu H, Wang W, Huang X, Yuan Z and Ai K: miR-212 promotes pancreatic cancer cell growth and invasion by targeting the hedgehog signaling pathway receptor patched-1. J Exp Clin Cancer Res 33: 54, 2014.

32. Tu H, Wei G, Cai Q, Chen X, Sun Z, Cheng C, Zhang L, Feng Y, Zhou H, Zhou B, et al: MicroRNA-212 inhibits hepatocellular carcinoma cell proliferation and induces apoptosis by targeting FOXA1. Onco Targets Ther 8: 2227-2235, 2015.

33. Ramalinga M, Roy A, Srivastava A, Bhattarai A, Harish V, Suy S, Collins S and Kumar D: MicroRNA-212 negatively regulates starvation induced autophagy in prostate cancer cells by inhibiting SIRT1 and is a modulator of angiogenesis and cellular senescence. Oncotarget 6: 34446-34457, 2015.

34. Borrego-Diaz E, Powers BC, Azizov V, Lovell S, Reyes R, Chapman B, Tawfik O, McGregor D, Diaz FJ, Wang X, et al: A potential regulatory loop between Lin28B:miR-212 in androgenindependent prostate cancer. Int J Oncol 45: 2421-2429, 2014. 
35. Zhao JL, Zhang L, Guo X, Wang JH, Zhou W, Liu M, Li X and Tang H: miR-212/132 downregulates SMAD2 expression to suppress the G1/S phase transition of the cell cycle and the epithelial to mesenchymal transition in cervical cancer cells. IUBMB Life 67: 380-394, 2015.

36. Liu H, Li C, Shen C, Yin F, Wang K, Liu Y, Zheng B, Zhang W, Hou X, Chen X, et al: miR-212-3p inhibits glioblastoma cell proliferation by targeting SGK3. J Neurooncol 122: 431-439, 2015.

37. Killick E, Morgan R, Launchbury F, Bancroft E, Page E, Castro E, Kote-Jarai Z, Aprikian A, Blanco I, Clowes V, et al: Role of Engrailed-2 (EN2) as a prostate cancer detection biomarker in genetically high risk men. Sci Rep 3: 2059, 2013.
38. Lee S, Jo H,Her J,Lee HY and Ban C: Ultrasensitive electrochemical detection of engrailed-2 based on homeodomain-specific DNA probe recognition for the diagnosis of prostate cancer. Biosens Bioelectron 66: 32-38, 2015.

39. Li Y, Liu H, Lai C, Su Z, Heng B and Gao S: Repression of engrailed 2 inhibits the proliferation and invasion of human bladder cancer in vitro and in vivo. Oncol Rep 33: 2319-2330, 2015.

40. Abollo-Jiménez F, Campos-Sánchez E, Toboso-Navasa A Vicente-Dueñas C, González-Herrero I, Alonso-Escudero E, González M, Segura V, Blanco O, Martínez-Climent JA, et al: Lineage-specific function of Engrailed-2 in the progression of chronic myelogenous leukemia to T-cell blast crisis. Cell Cycle 13: 1717-1726, 2014 